\title{
Ground Penetrating Radar: water table detection sensitivity to soil water retention properties
}

\author{
Albane Saintenoy and Jan W. Hopmans
}

\begin{abstract}
We are interested in Ground Penetrating Radar (GPR) as a geophysical tool useful for determining the depth of the ground water table (GWT) and for monitoring shallow water infiltration in sandy soils. At hydrostatic equilibrium, the water content distribution in a homogeneous unsaturated soil down to the water saturated zone depends on the soil water retention function. A classical way to fit retention curve data is to use the van Genuchten continuous model. Using Finite Difference Time Domain simulations, we study the sensitivity of the GPR signal reflected by a van Genuchten type transition to the hydraulic parameters. We show a power type relationship between the reflected signal amplitude and the slope of the soil retention curve. Furthermore, for simulating GPR reflection data acquired above a transition from unsaturated to saturated soil, geophysicists often approximate the soil water retention curve by a piece-wise linear model. We test the validity of such an approximation depending on the frequency of the radar signal and the abruptness of the retention curve. We illustrate our results with high resolution GPR data $(1600 \mathrm{MHz})$ acquired above a fluctuating water table in a sand column at the laboratory scale.
\end{abstract}

\section{INTRODUCTION}

$\mathbf{S}$ OIL water content and its depth distribution are determined in part by the soil hydraulic properties. Specifically, the soil water retention curve relates the soil water pressure head, $h$, to its volumetric water content $\theta$, and its shape is determined by soil texture and pore size distribution. By definition, the groundwater table (GWT) depth corresponds to the soil depth where the soil water pressure $h=0$, and $\theta$ is close to the saturated water content $\theta_{s}$. The capillary fringe defines the distance above the GWT where the soil remains saturated, despite the soil water pressure being below atmospheric pressure. The value of $h$ at which the soil desaturates is defined as the air-entry value. As $h$ decreases further, $\theta$ decreases, following to a S-shaped curve with an inflection point, towards a soil-specific water content known as the residual water content $\theta_{r}$. More details on retention curve and the most commonly used parametric models that are available to fit soil water retention data are available in [1]. The air/water capillary transition zone (CTZ) is defined as the region of sharp decrease of $\theta$ from $\theta_{s}$ to $\theta_{r}$ directly above the capillary fringe (Fig. 1). In sandy soils, the transition zone thickness varies from 10 to $100 \mathrm{~cm}$ depending on the grain size distribution. Such a narrow transition zone can not be

Albane Saintenoy is with the UMR 8148 Interaction et Dynamique des Environnement de Surface, Université Paris Sud 11, Faculté des Sciences, 91405 Orsay, France, and the CNRS, e-mail: (albane.saintenoy@u-psud.fr).

Jan W. Hopmans is with the Department Land Air Water Resources, University of California at Davis, CA 95616, USA, e-mail: (jwhopmans@ucdavis.edu). identified for finer-textured soils where pore size distributions are much more gradual.

GPR reflection measurements have been used succesfully to image GWT variations underground in [2], [3], [4], [5] and [6]. The GPR reflectivity of a transition zone above a GWT was discussed in [7], [8] and [9]. Using two-dimensional numerical model simulations of electromagnetic field propagation in soils above a GWT, [7] determined that a GWT can be best detected if the dominant wavelength of the GPR signal is more than 3 times the transition zone thickness. In their study, they used a sinus function to describe the gradual transition between residual and saturated water content in the transition zone. Similarly, using a linear model for the CTZ, [8] concluded that "when the dominant wavelength of the GPR antenna is very long compared to the thickness of the transition layer, its presence is clearly of no importance".

Those studies are in contradiction with results from [9], [6] and [10]. In [9], the authors investigated numerically the 1 GHz GPR signal reflected by an approximately $1 \mathrm{~m}$ thickness CTZ. They also validated their study with some field data for which borehole data were available along the GPR acquisition line. Their conclusion is that the GPR signal reflected by the CTZ does not occur from the phreatic water level, where $h=0$, but appears to come from the depth level where the capillary pressure-water saturation curves show maximum curvature. They used the empirical expression from Brooks and Corey [11] to model the saturation dependence of the soil water pressure that includes a discontinuity in the first derivative at the air-entry point.

Application of surface GPR to monitor water injection into a sandy soil using a $800 \mathrm{MHz}$ antenna was described in [6]. Since the transition zone of this sandy soil was approximately $25 \mathrm{~cm}$, the GPR wavelength was about two third of the transition zone thickness, assuming a background velocity of $0.1 \mathrm{~m} / \mathrm{ns}$. Though this was not in full agreement with the recommended relation in [7] and [8], reflections associated with soil water content variations appeared clearly in [6].

Lambot et al [10] showed a controlled field study where they were able to reconstruct the water retention curve by full waveform inversion of monostatic off ground GPR data. They demonstrated that GPR data with a frequency bandwidth from $800 \mathrm{MHz}$ to $2.8 \mathrm{GHz}$ contain sufficient information to detect the water table. They performed full waveform inversion to find the water retention curve parameters using the Mualem van Genuchten model. However, trying a simplified threelayered dielectric profile, large errors are observed for the second and the third layers. They attributed those errors to high noise in the measurements and to the simplifying assumptions 


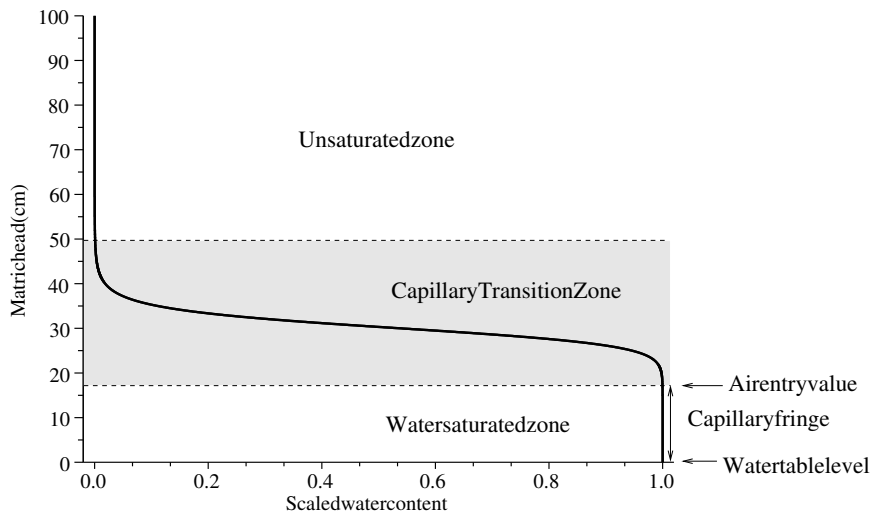

Fig. 1. Theoretical van Genuchten function and its main features.

in this model.

With these preceding studies in mind, we present in this paper numerical experiments to investigate the relation between a high frequency GPR reflected signal and the retention curve characteristics of a medium-textured sand modeled by a van Genuchten model. We also study the effect of using a simplified piece-wise linear model on the GPR reflected signal. We will confirm our results with experimental data acquired with a $1600 \mathrm{MHz}$ antenna above a changing GWT in a $60 \mathrm{~cm}$ tall and $45 \mathrm{~cm}$ diameter sand column.

\section{Material And Methods}

\section{A. The Van Genuchten Retention Curve}

The most common parametric model used to fit soil water retention data is the van Genuchten function [12],

$$
\Theta=\frac{\theta-\theta_{r}}{\theta_{s}-\theta_{r}}=\left(\frac{1}{1+(\alpha|h|)^{n}}\right)^{1-\frac{1}{n}},
$$

where $\Theta$ is the normalized soil water content, $\theta$ is the equilibrium water content $\left(\mathrm{cm}^{3} \mathrm{~cm}^{-3}\right)$ for a given soil water matric head $h(\mathrm{~cm})$ at any point in the soil column, $\theta_{r}$ and $\theta_{s}$ are the residual and saturated water content values, respectively, and $\alpha(1 / \mathrm{m})$ and $n$ are fitting parameters. The parameter $\alpha$ has an effect on the air-entry value. The parameter $n$ is related to the slope of the transition zone. High $n$ values imply small transition thickness. We evaluate its effect on the GPR reflection coming from a GWT with the following numerical simulations.

\section{B. The Dielectric Mixing Model}

In order to simulate the GPR signal propagating through a material with a given retention curve above the GWT, a relation between the dielectric permittivity $\varepsilon$ and the soil water content $\theta$ is needed. For that purpose, we assumed the dielectric mixing model of [13] to be valid. In this model, the bulk permittivity of an isotropic soil-water-air soil system, $\varepsilon$, is expressed by the Complex Refractive Index Model, or

$$
\varepsilon=\left(\theta\left(\sqrt{\varepsilon_{w}}-1\right)+(1-\phi) \sqrt{\varepsilon_{s}}+\phi\right)^{2},
$$

where $\phi$ is the estimated soil porosity, and $\varepsilon_{w}$ and $\varepsilon_{s}$ are the dielectric permittivity values of water and soil particles.



Fig. 2. Retention curves whose characteristic are given in Table I.

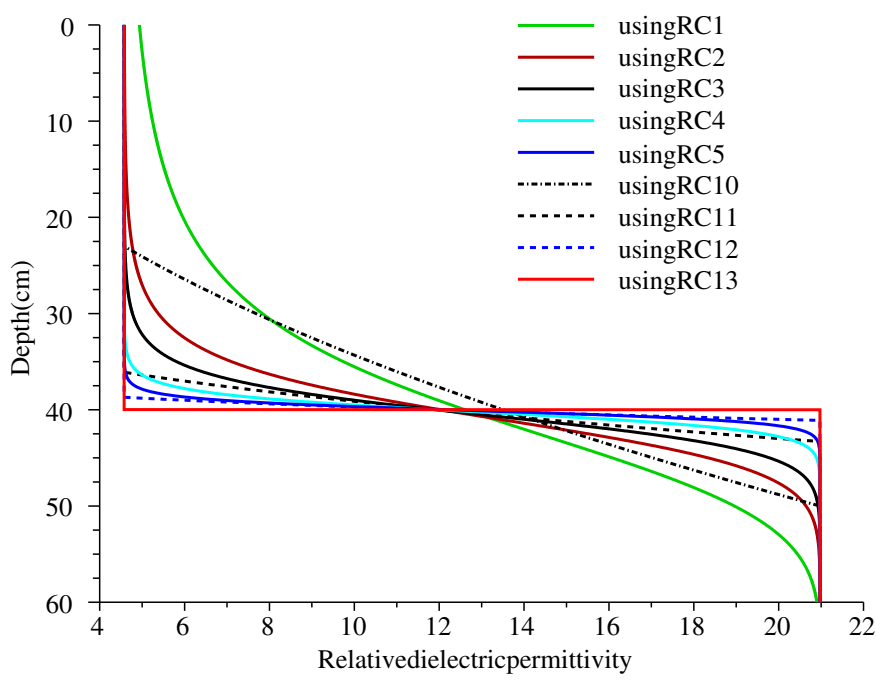

Fig. 3. Relative dielectric permittivity profiles used to simulate the traces of Fig. 4, 5, 7 and 8 . The water table is $70 \mathrm{~cm}$ deep.

Further on, all dielectric permittivity values are given relative to its value in free space, that is $8.85410-12 \mathrm{~F} / \mathrm{m}$. We use $\varepsilon_{s}=3.89$ as the dielectric constant for sand, as reported by [14], and $\varepsilon_{w}=80.1$ as the dielectric constant value of water at $20 \mathrm{C}$. In all of our analysis, we assume that changes in magnetic permeability are negligible, by setting the relative magnetic permeability to 1.0 for all simulations. We set electrical conductivity to be $0 \mathrm{~S} / \mathrm{m}$.

\section{Numerical Modeling}

Electromagnetic simulations were conducted using GprMax2d [15]. This numerical code uses a finite difference scheme to simulate the propagation of an electromagnetic wave in two or three dimensions. We restricted the modeling to two dimensions only. The radar source was modeled by the classical Ricker waveform (second derivative of a Gaussian function), centered at $1100 \mathrm{MHz}$ (central frequency of the experimental data presented at the end of this paper). 
TABLE I

RETENTION CURVE CHARACTERISTICS (VG: VAN GENUCHTEN; PL: PIECEWISE LINEAR).

\begin{tabular}{|c|c|c|c|c|c|}
\hline Name & Curve type & \multicolumn{4}{|c|}{ Parameters } \\
\hline & & $\theta_{r}$ & $\theta_{s}$ & $\alpha\left(\mathrm{m}^{-1}\right)$ & $n$ \\
\hline $\mathrm{RC} 1$ & $\mathrm{vG}$ & 0.065 & 0.372 & 3.32 & 5 \\
\hline $\mathrm{RC} 2$ & $\mathrm{vG}$ & 0.065 & 0.372 & 3.32 & 10 \\
\hline RC3 & $\mathrm{vG}$ & 0.065 & 0.372 & 3.32 & 15 \\
\hline $\mathrm{RC} 4$ & $\mathrm{vG}$ & 0.065 & 0.372 & 3.32 & 30 \\
\hline RC5 & $\mathrm{vG}$ & 0.065 & 0.372 & 3.32 & 50 \\
\hline RC6 & $\mathrm{vG}$ & 0.065 & 0.372 & 3.32 & 75 \\
\hline RC7 & $\mathrm{vG}$ & 0.065 & 0.372 & 3.32 & 100 \\
\hline RC8 & $\mathrm{vG}$ & 0.065 & 0.372 & 3.32 & 150 \\
\hline RC9 & $\mathrm{vG}$ & 0.065 & 0.372 & 3.32 & 200 \\
\hline & & $\theta_{r}$ & $\theta_{s}$ & $h_{1}(\mathrm{~cm})$ & $h_{2}(\mathrm{~cm})$ \\
\hline $\mathrm{RC} 10$ & PL & 0.065 & 0.372 & 20 & 47 \\
\hline$\overline{R C 11}$ & $\overline{\mathrm{PL}}$ & 0.065 & 0.372 & 26.7 & 33.9 \\
\hline $\mathrm{RC} 12$ & PL & 0.065 & 0.372 & 28.9 & 31.3 \\
\hline $\mathrm{RC} 13$ & PL & 0.065 & 0.372 & 30 & 30 \\
\hline
\end{tabular}

All GPR simulations were conducted using a spatial mesh of $2 \mathrm{~mm}$ squared grids for a $2.2 \mathrm{~m}$ wide and $2.6 \mathrm{~m}$ height soil domain. The computational time step used was $4.710^{-12} \mathrm{~s}$. The model domain consisted of a $2 \mathrm{~m}$ thick air layer, above a $60 \mathrm{~cm}$ deep soil, using 301 soil layers of $2 \mathrm{~mm}$ thickness each. The bottom of the model domain included a $2 \mathrm{~mm}$ thick Perfect Electrical Conductive layer. The strong reflection on this layer will be used as a marker of the bottom of the model. Simulations were conducted using dielectric values derived from water content variations using (2), assuming a water content distribution resulting from hydraulic equilibrium with a GWT depth of $70 \mathrm{~cm}$. Results were compared using various soil water retention functions ( $\mathrm{RC} 1$ to $\mathrm{RC} 13$ in Table I) displayed on Fig. 2. The dielectric permittivity variations in depth are displayed in Fig. 3.

\section{SENSITIVITY ANALYSIS}

\section{A. Sensititivy to the parameter $n$}

Differences between retention curves RC1 to RC9 (Table I) are determined by the $n$ value. The parameter $n$ varies from 5 in RC1 up to 250 in RC9. The value of $n$ affects the abruptness of the transition zone as illustrated in Fig. 2. The higher $n$ is, the closer the retention curve is similar to the step function RC13. The traces simulated assuming a GPR acquisition above a $60 \mathrm{~cm}$ thick soil presenting those retention curves (GWT depth at $70 \mathrm{~cm}$ ) are shown on Fig. 4. No gain is applied to allow for amplitude comparison between traces. The velocity is inversely proportional to the square-root of the permittivity. The arrival time of the reflection on the underlying PEC layer varies depending on the retention curve used to create the permittivity profile, but there is no major amplitude differences. The time delay would be much more sensitive to $\left(\theta_{s}-\theta_{r}\right)$ and $\alpha$.

As we are interested in the reflection of the transition zone, we window the part of the data between 4 and 10 ns (Fig. 5). We normalize the traces to obtain the same amplitude as for

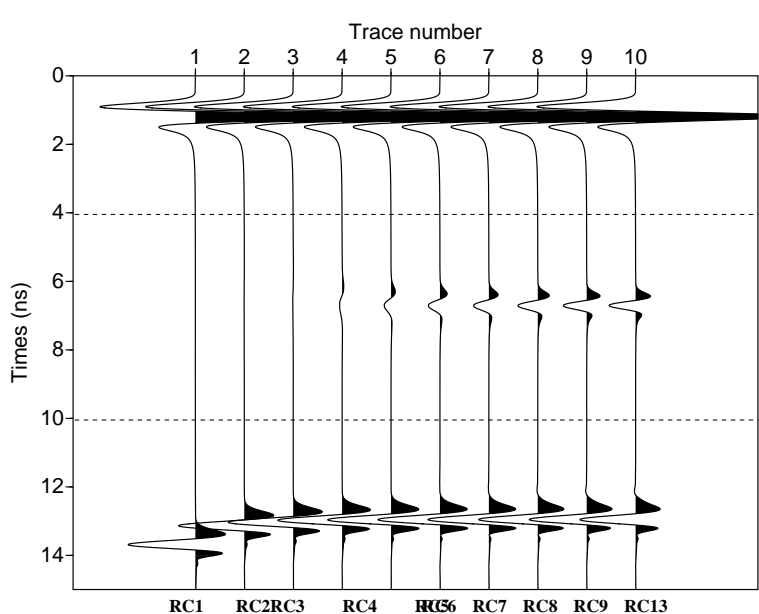

Fig. 4. Simulated traces resulting from retention curve models RC1 (trace 1) to RC9 (trace 9) and RC13 (trace 10) as described on Table I.

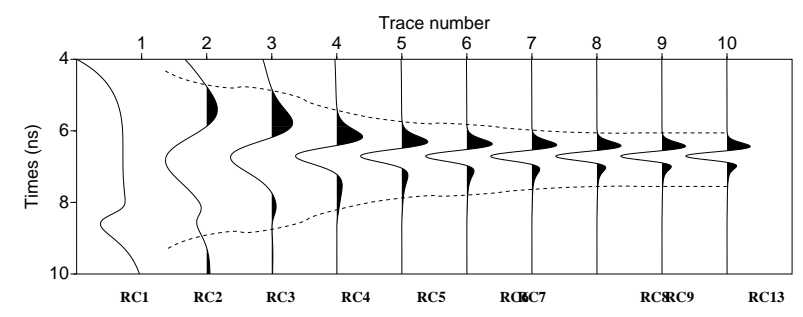

Fig. 5. Close-up on the reflection coming from the transition zone for simulated traces of Fig. 4. Each trace has been normalized to the maximum reflected amplitude when using the step function $\mathrm{RC} 13$. In traces 1 and 2, the signal is blurred by the noise.

the step function RC13 (trace 10). This scaling emphasizes the shape of the reflection of the transition zone for small values of $n$. The reflected signal is wider as the parameter $n$ is smaller (Fig. 5). The arrival time of the minimum trough is equal in all simulated traces except when using RC1 due to the noise. Knowing the velocity distribution from permittivity profiles, we can convert our arrival time to depth and show that the trough corresponds to the inflection points of all used van Genuchten curves, corrobating Nguyen et al.'s conclusion [9]. This can be explained by considering the reflections as the convolution of the reflectivity curve and the source signal.

We called $A_{n}$ the ratio between the amplitude of the reflection for a given retention curve and the amplitude for the step function RC13. In order to highlight the effect of $n$ on the amplitude of the reflection, Fig. 6 shows the ratio $A_{n}$ in relation with the parameter $n$. For $n<50$, as measured in most soils, the relation is fitted by an exponential function as

$$
A_{n}=n^{2} 10^{-4} \text {. }
$$

The amplitude of the GPR signal reflected on the CTZ is less than a third of the one created by the step function. This explains why [7] and [8] conclude that the GPR signal wavelength should be higher than the CTZ thickness for best GWT imaging. However it does not mean that this reflected 


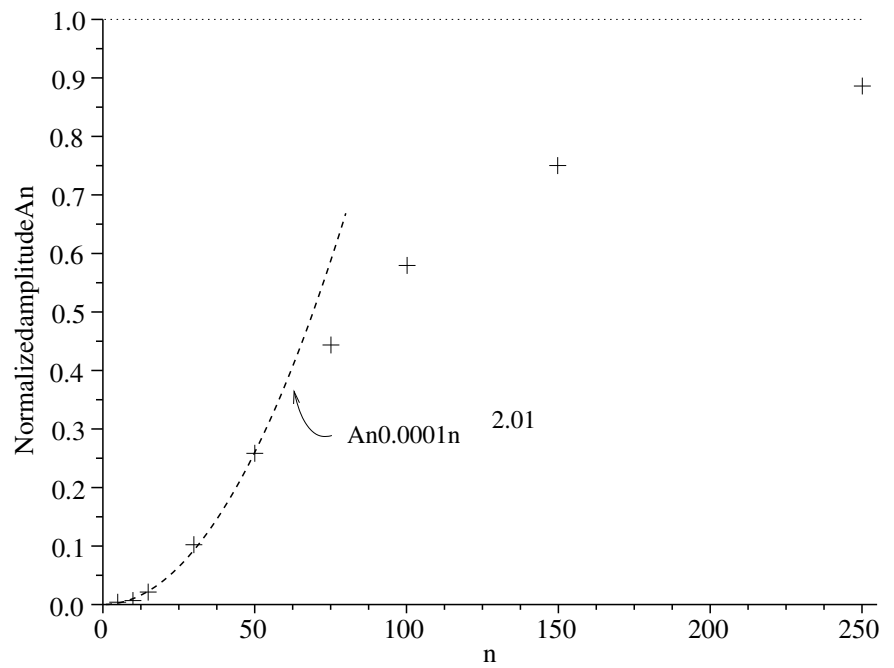

Fig. 6. Relation between the maximum amplitude of the reflection (normalized with the maximum amplitude obtained with a step function RC13) and n.

signal does not exist or that the wave has been attenuated in the unsaturated zone as said in [10].

\section{B. Piecewise Linear Model Approximations}

For simulating GPR reflection data acquired above a transition from unsaturated to saturated soil, geophysicists approximate the soil water retention curve by a piece-wise linear model as in [8]. To test the validity of such an approximation, we considered two different piece-wise linear models to describe the transition zone. In the first model, we considered a linear decrease of the water content from $\theta_{s}$ down to $\theta_{r}$ when $h$ varies from its air-entry value down to its value when the soil has a residual water content. Using this approach, the van Genuchten retention curve RC3 has been approximated by RC10 (Fig. 2). A second model consisted in taking the linear transition piece tangent to the S-shape transition zone (equaling the slope of the linear decrease and the derivative of the S-shape curve at its inflection point). With this method, RC3 was approximated by RC11 and RC5 by RC12 in Fig. 2 .

The resulting simulated traces are displayed in Fig. 7 (with no gain) and a close-up is on Fig. 8 (using a normalization to equal the reflection on the step function). Clearly, traces 1 , 2 and 3 are different. Each change of slope in the piece-wise models is creating a reflection in the GPR data. This is not the case when considering a van Genuchten curve.

The piece-wise linear model is improving when considering RC5 with $n=50$. The transition thickness associated with RC5 is small enough in comparison with the GPR signal wavelength and the shape of the reflected signal becomes similar between traces 4,5 and 6 of Fig. 8. There remains a difference in the amplitude (Fig. 7).

\section{LABORATORY EXPERIMENTS}

A fluctuating water table experiment was conducted in a $45-\mathrm{cm}$ diameter and $60-\mathrm{cm}$ tall polyvinyl chloride (PVC) column. The column was filled with a 30 mesh Monterey sand

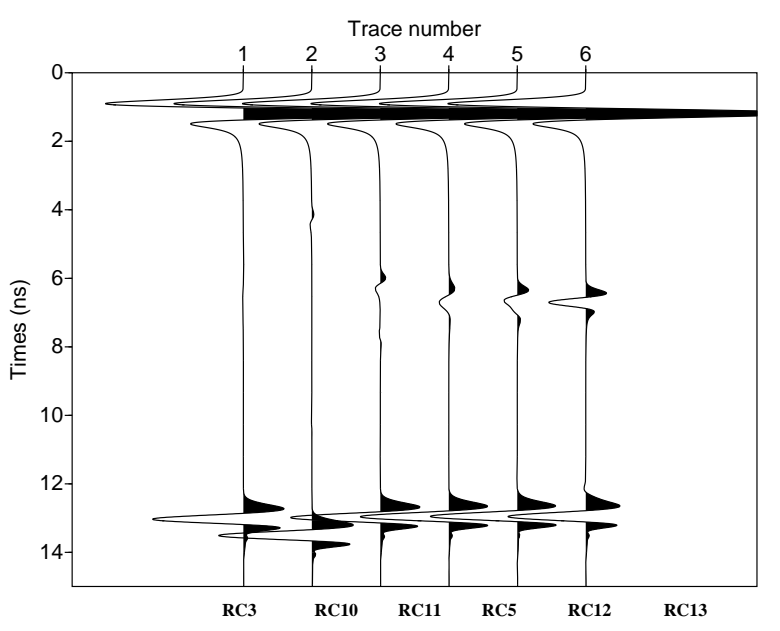

Fig. 7. Simulated traces obtained using a model derived from retention curves RC3 and RC5 (Table I) approximated by linear functions (RC10 to $\mathrm{RC} 13$ in Table I).

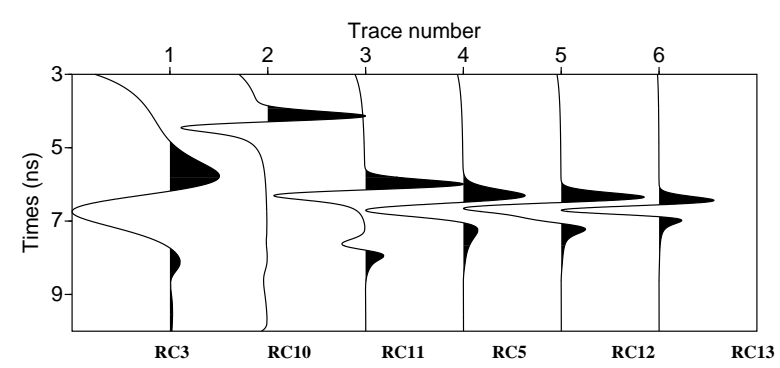

Fig. 8. Close-up on the reflections coming from the transition zone in Fig. 7. Each trace has been normalized to the maximum reflected amplitude when using the step function RC13.

(RMC, Natural Monterey Beach Sand, Cemix, Pleasonton, CA 94566), that is typically used for sandblasting purposes. Based on sieve analysis results, it was determined that $83 \%$ of the sand grains were between $0.25 \mathrm{~mm}$ and $0.5 \mathrm{~mm}$. The column was placed on a laboratory bench in a constant temperature room at about $20 \mathrm{C}$. Prior to filling the column, we installed interconnected porous sintered glass tubes at the bottom of the tank, allowing GWT depth control. A separate porous tube was placed at the bottom and connected to a water manometer, to ensure hydraulic equilibrium between changing GWT levels.

To support our work, we show in Fig. 9 the recorded traces by a Mala Ramac $1600 \mathrm{MHz}$ antenna placed on top of the sand column for different GWT depths. Fig. 9 compares measured (near column wall) with simulated traces (RC3) for 15 GWT steps. For best visualisation, each trace is normalized to its maximum amplitude, amplitudes are clipped to $90 \%$ of their maximum amplitude and the real traces are scaled by 0.3 .

As the GWT depth increases, the water content inside the column decreases and the average travel velocity across the column increases, as attenuation is decreasing. Therefore, the reflection at the PEC layer (encircled with green dashes) is arriving earlier with a higher amplitude for the deeper GWT 


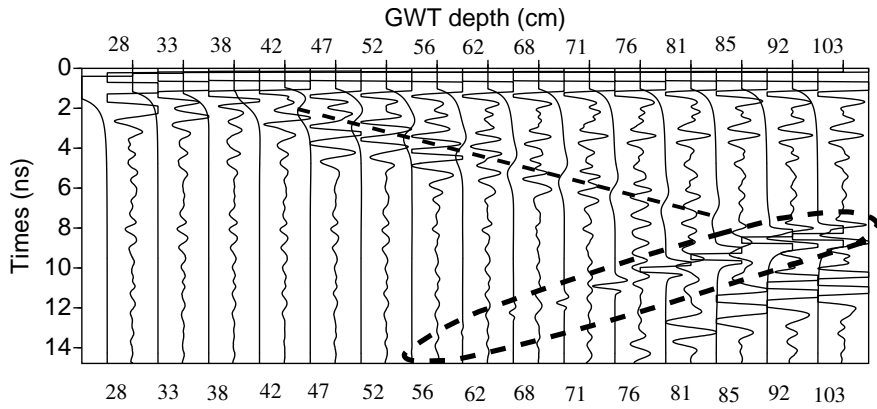

Fig. 9. Comparison at each GWT depth between 2D simulated traces using RC3 (first trace) and measured traces in a laboratory experiment (second trace). The dashed line links the main trough of the synthetic reflections on the transition zone. The dashed ellipse encircles the reflection on the PEC interface.

depths. When the GWT depth varies from 47 to $85 \mathrm{~cm}$, we detect the signal trough for the simulated traces with corresponding delay times ranging between $2 \mathrm{~ns}$ to $8 \mathrm{~ns}$. Knowing the model used for the simulation, it was confirmed that the arrival time of the troughs corresponds to the time needed to travel from the surface to the inflection point of the water content distribution, as determined by the soil water retention curve. Even with the low signal to noise ratio in the real data, each position of the reflection on the simulated traces correlates to a zone of higher amplitude in the real data.

\section{CONCLUSION}

When acquiring GPR data above a sandy soil with a shallow GWT, the retention curve plays a significant role. The source of the GPR reflection event is the variation in dielectric permittivity that occurs above the GWT. In contrast, the GWT is defined as the depth where the pressure head is zero. These two features can only be connected through knowledge of the soil water retention properties such as the van Genuchten parameters $\alpha, n, \theta_{r}$ and $\theta_{s}$.

Using Finite Difference Time Domain simulations with a Ricker wave centered on $1100 \mathrm{MHz}$, we studied the sensitivity of the GPR signal reflected by the van Genuchten type transition to the hydraulic parameters. We show a power type relationship between the reflected signal amplitude and the $n$ parameter. The effect of the $n$ parameter on the amplitude of the reflection above a transition from water unsaturated to saturated sand demonstrates that a GWT is not always detectable by using high frequency GPR, especially for finertextured soils such as loams and clays.

When the CTZ thickness is of the same order as the wavelength of the sounding wave, piecewise linear approximation of the retention curve is misleading and may cause an inaccurate GWT depth estimation. Each change of slope in the piece-wise models is creating a reflection in the GPR data and the resulting signal is the sum of those delayed reflections. When the CTZ thickness is small enough compare to the probing signal wavelength, the difference in the reflected signal from piece-wise linear, van Genuchten or step models is diminishing.

When detectable, the maximum of the reflected signal envelope is related to the position of the inflection point of the S-shape transition curve. The retention curve needs to be estimated to allow detection of the GWT, relatively to the position of the inflection point. The amplitude of the reflection is highly sensitive to $n$, but the water content range $\left(\theta_{s}-\theta_{r}\right)$ and the parameter $\alpha$ must be estimated independently from the GPR reflected signal. The laboratory experiment confirms the simulation results, when using a $1600 \mathrm{MHz}$ antenna for a sandy soil with a transition zone thickness that is of the same magnitude as the GPR wavelength.

\section{ACKNOWLEDGMENT}

The authors would like to thank warmly J. Mc-Intyre, A. Tuli, T. Kamai, F. Kizito, and A. Malazian for their help for setting up the laboratory experiments, as well as P. Tucholka and H. Zeyen for their enlightening discussions, and Evert Slob and one anonymous reviewer for their constructive comments.

\section{REFERENCES}

\section{REFERENCES}

[1] K. Kosugi, J. H. Dane, and J. W. Hopmans, Parametric models. Soil Science Society od America, Inc., 2002, pp. 739-755.

[2] G. Vellidis, M. C. Smith, D. L. Thomas, and L. E. Asmussen, "Detecting wetting front movement in a sandy soil with ground penetrating radar," Transactions of the ASAE, vol. 33, no. 6, pp. 1867-1874, 1990.

[3] Y. Nakashima, H. Zhou, and M. Sato, "Estimation of groundwater level by gpr in an area with multiple ambiguous reflections," Journal of Applied Geophysics, vol. 47, pp. 241-249, 2001.

[4] A. L. Endres, W. P. Clement, and D. L. Rudolph, "Ground penetrating radar imaging of an aquifer during a pumping test," Ground Water, vol. 38, pp. 566-576, 2000.

[5] M. J. Bevan, A. L. Endres, D. L. Rudolph, and G. Parkin, "The noninvasive characterization of pumping-induced dewatering using ground penetrating radar," Journal of Hydrology, vol. 281, pp. 55-69, 2003.

[6] A. Saintenoy, S. Schneider, and P. Tucholka, "Evaluating ground penetrating radar use for water infiltration monitoring," Vadose zone journal, vol. 7, pp. 208-214, 2008.

[7] A. P. Annan, S. W. Cosway, and J. D. Redman, "Water table detection with ground-penetrating radar," in Society of Exploration Geophysics Technical Program Expanded Abstracts, 1991, paper E/G1.4, pp. 494 496.

[8] M. Bano, "Effects of the transition zone above a water table on the reflection of GPR waves," Geophysical Research Letters, vol. 33, p. L13309, 2006.

[9] B.-L. Nguyen, J. Bruining, E. C. Slob, and V. Hopman, "Delineation of air/water capillary transition zone from GPR data," SPE Reservoir Evaluation \& Engineering, pp. 319-327, 1998.

[10] S. Lambot, J. Rhebergen, I. van den Bosh, E. C. Slob, and M. Vanclooster, "Measuring the soil water content profile of a sandy soil with an off-ground monostatic ground-penetrating radar," Vadose Zone Journal, vol. 3, pp. 1063-1072, 2004.

[11] R. H. Brooks and A. T. Corey, "Hydraulic Properties of Porous Media," Hydrology Papers, Colorado State University, no. 3, 1964.

[12] M. T. V. Genuchten, "A closed form equation for predicting the hydraulic conductivity of unsaturated soils," Soil Sci. Soc. Am. J., vol. 44, pp. 892$898,1980$.

[13] J. R. Birchak, C. G. Gardner, J. E. Hipp, and J. M. Victor, "High dielectric constant microwave probes for sensing soil moisture," in Proceedings of the IEEE, vol. 62, 1974, pp. 93-98.

[14] A. Alharthi and J. Lange, "Soil water saturation: dielectric determination," Water Resources Research, vol. 23, no. 4, pp. 591-595, 1987.

[15] A. Giannopoulos, "Modelling ground penetrating radar by gprmax," Construction and building materials, vol. 19, no. 10, pp. 755-762, 2005. 
Albane Saintenoy Albane Saintenoy was born in Angers, France, in 1970. She received the Ph.D. degree in Geophysics in 1998, from the University Denis Diderot - Paris 7, France. Her Ph.D. work was on the electromagnetic parameters characterization from multi-offset acquisition of ground-penetrating radar (GPR) data.

From 1998 to 2000, she was a post-doctoral fellow at the Center for Wave Phenomena, Colorado School of Mines, USA, working on the inversion of borehole vertical profiling radar data. In 2000, she joined the Earth Science department, University Paris Sud, France, where she is currently an Associate Professor. In 2008, she spent a sabbatical year at the Land Air Water Resource Department of the University of California in Davis. Her current research interests are soil hydraulic parameters determination from surface GPR measurements, joined GPR data and electrical resistivity measurements for cavity detection, and frozen soil GPR soundings.
Jan W. Hopmans Jan Hopmans received the M.Sc. degree in 1981 from Wageningen Agricultural University, The Netherlands, and the Ph.D degree in Soil Physics in 1985 from the Auburn University, USA. In 1988, he joined the Department of Land, Air and Water Resources, University of California, Davis, CA, where he is currently a Professor in the Hydrology Program.

Among different honors, he received the Don and Betty Kirkham Soil Physics Award in 2003 and is a Fellow American Geophysical Union since 2005. His current research interests are related to vadose zone hydrology, specifically soil physics, unsaturated flow and transport processes, contaminant hydrology, x-ray microtomography, scaling techniques, plant root-soil water interactions, parameter optimization, soil hydraulic properties, flow and transport modeling and irrigation water management. 